\title{
Iterative Refinement of Point Correspondences for 3D Statistical Shape Models
}

\author{
Sharmishtaa Seshamani, Gouthami Chintalapani, and Russell Taylor \\ Department of Computer Science, Johns Hopkins University, Baltimore, MD
}

\begin{abstract}
Statistical atlases of bone anatomy are traditionally constructed with point-based models. These methods establish initial point correspondences across the population of shapes and model variations in the shapes using a variety of statistical tools. A drawbacks of such methods is that initial point correspondences are not updated after their first establishment. This paper proposes an iterative method for refining point correspondences for statistical atlases. The statistical model is used to estimate the direction of "pull" along the surface and consistency checks are used to ensure that illegal shapes are not generated. Our method is much faster that previous methods since it does not rely on computationally expensive deformable registration. It is also generalizable and can be used with any statististical model. We perform experiments on a human pelvis atlas consisting of 110 healthy patients and demonstrate that the method can be used to re-estimate point correspondences which reduce the hausdorff distance from $3.2 \mathrm{~mm}$ to $2.7 \mathrm{~mm}$ and the surface error from $1.6 \mathrm{~mm}$ to $1.4 \mathrm{~mm}$ for PCA modelling with 20 modes 1
\end{abstract}

\section{Introduction}

Statistical atlas modelling is a popular tool for analysis of several types of medical images. Applications range from modelling of anatomical variations within shape populations to pathological anomaly detection. Shape atlases are typically built by selection of a representation such as points [1,2, 3], curves [4] or level sets [5] followed by modelling using various statistical techniques 6 . In this paper, we address the application of statistical bone atlases. Traditionally, bone (and other rigid body) atlases are modelled using dense point-based methods coupled with linear statistical models. A set of shapes are first represented as either surface meshes or volumetric tetrahedral meshes. Subsequently, vertex correspondences are established using a template mesh. These vertices are then used to represent the shapes as high dimensional vectors which can be statistically analyzed for shape variation and other applications.

One drawback of this approach is that point correspondences are established beforehand and never updated once the model is built. As a consequence, the error and uncertainty of this initialization is propagated through the model. Chintalapani et al [2] propose a solution for updating the model with a bootstrapping method which employs the intensity values of voxels. However, this method

${ }^{1}$ Supported by NIH with Grant 1-R01-EB00683 and JHU internal funds. 
is computationally very expensive since it requires multiple passes through an intensity-based deformable registration algorithm. Secondly, in some cases, the 3D intensity data may not be available. In more recent work, Davies et al [3] propose a minimum description length framework for optimizing a PCA based shape model. Although this method implicitly disallows generation of illegal shapes, it is also computationally very expensive. Secondly, their method is specific to PCA based modelling. In this paper, we propose an iterative bootstrapping method for improving point correspondences without any outside information with the use of any statistical model. A set of initial point correspondences are established (just as in most prior work) to estimate a statistical model, which is then used to re-estimate point correspondences. This iterative procedure can be repeated until convergence. We describe the method for the re-estimation with a PCA based model. However, our method can be applied to any generic statistical model. Leave-out validation and comparison of the vertex error, surface error and volume difference is carried out. Results show that our method is able to improve modelling performance with all these metrics.

\section{Methods}

We begin with a set of $N$ surface meshes described as: $\mathcal{S}=\left\{S_{i}=\left(T, V_{i}\right) \mid i=\right.$ $1 \ldots N\}$, where $V_{i} \in \mathcal{R}^{P X 3}$ contains the $P$ vertices of the shape and $T$ is the set of triangles. The set $\mathcal{V}=\left\{V_{1} \ldots V_{N}\right\}$ contains initial point correspondences, ie: the $n$th vertices of each shape correspond. The objective is to generate a statistical model of this point (vertex) based representation and then improve the initial point correspondences. In the next few sections, we define a few preliminaries before describing the general algorithm.

\subsection{Preliminaries}

Given the set $\mathcal{V}$, we select one test sample $V_{i}$. The rest of the samples form a set $\mathcal{V}^{\prime}{ }_{i}$ which is used to generate a PCA model consisting of a mean shape $\bar{M}$ and the modes of variation $Y$. $V_{i}$ can now be represented by a set of mode weights $\lambda$, computed as: $\lambda=Y^{T}\left(V_{i}-\bar{M}\right)$. The mode weights can be used to reconstruct the test sample as: $V_{i}^{r e c}=\bar{M}+\sum_{i=1}^{n} \lambda_{i} Y_{i}$. This type of leave-out modelling and reconstruction can be performed for all $V_{i} \in \mathcal{V}$. Figure 1(a) shows an example of an original shape and some points on it in red $\left(V_{i}\right)$. The grey points are those that are reconstructed using the model $\left(V_{i}^{r e c}\right)$.

Reconstruction with leave-one-out modelling produces an estimate of the points on the left out shape using a model generated by all the other shapes. For each vertex point, the vector relating the original point to the reconstructed points has two components: an out of surface component $\boldsymbol{d}_{\text {out }}$ and an along surface component $\boldsymbol{d}_{\text {along }}$ (Figure 1(b)]. In order to ensure point correspondence update only along the surface, we can compute the closest point on the original surface to the reconstructed point (Figure 1(a). This now gives us the direction that the statistical model "pulls" the original points along the surface. Carrying this out for all vertex points in $V_{i}$ generates a set of 
vertices $V_{i}^{\text {closest }}$. In some cases, the closest point may move very far away from the original point. Update with the closest point could therefore generate large amounts of distortion in the shapes and collapsing of triangles. Instead of performing a closest point update, we can now move the original point in the direction of the closest point along the surface by applying a scale factor $\mu . \mu=1$ generates the closest point itself. However, this may not always correspond to a consistent shape. Hence, we can select a $\mu$ which does satisfy this requirement. Once $\mu$ is selected, the point can be moved by the vector $\mu \boldsymbol{d}_{\text {along }}$ and then projected back onto the surface (closest point to the surface) to generate the updated points $V_{i}^{\text {updated }}$ (Figure 1(c)). In the experimental section, we show how the fractional updates preserve consistency and reduce shape distortion for improved point correspondences. Modifying the vertices of a surface mesh can affect the consistency of the reconstruction. In particular, we are interested in avoiding generation of illegal shapes. For mesh based representations, comparison of the normals of the triangles in the original and updated shapes gives a quantitative measure of illegal shapes (flipped triangles) generation. Given a surface $S_{i}=\left(V_{i}, T\right)$ and the new surface $S_{i}^{\text {updated }}=\left(V_{i}^{\text {updated }}, T\right)$, the normals of all triangles of $S_{i}$ and $S_{i}^{\text {updated }}$ can be computed to generate the sets $N_{i}$ and $N_{i}^{\text {updated }}$ respectively. The dot products of normals of corresponding triangles can be computed. If a triangle has been flipped, the dot project will be negative.

\subsection{Algorithm Summary}

Algorithm 1 and Figure 2 outline the procedure. Initial set of point correspondences are iteratively updated until convergence. In each iteration, the input set of surfaces is $\mathcal{S}^{\text {updated }}$ which was generated by the previous iteration. Closest points are always computed to the original set of surfaces $\mathcal{S}$ in order to maintain the shape of the surfaces.

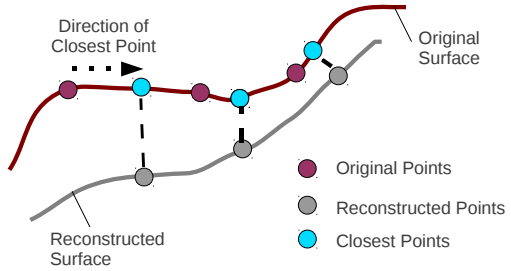

(a) Original surface with a few points marked (Red), Surface reconstructed with PCA with points marked (Grey), Closest Points (Blue) and direction of closest point from original point

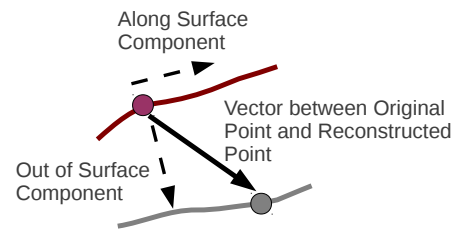

(b) Out of Surface and Along Surface Components of vector between original and reconstructed point

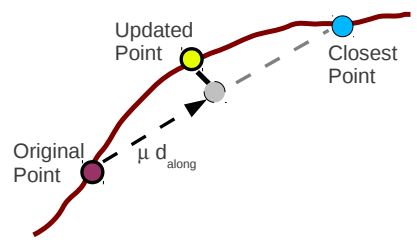

(c) Fractional Update of Points: The point is moved in the direction of the closest point at a scale of $\mu$ and then projected onto the surface to generate the updated point (correspondence).

Fig. 1. Explanatory figures for preliminaries 

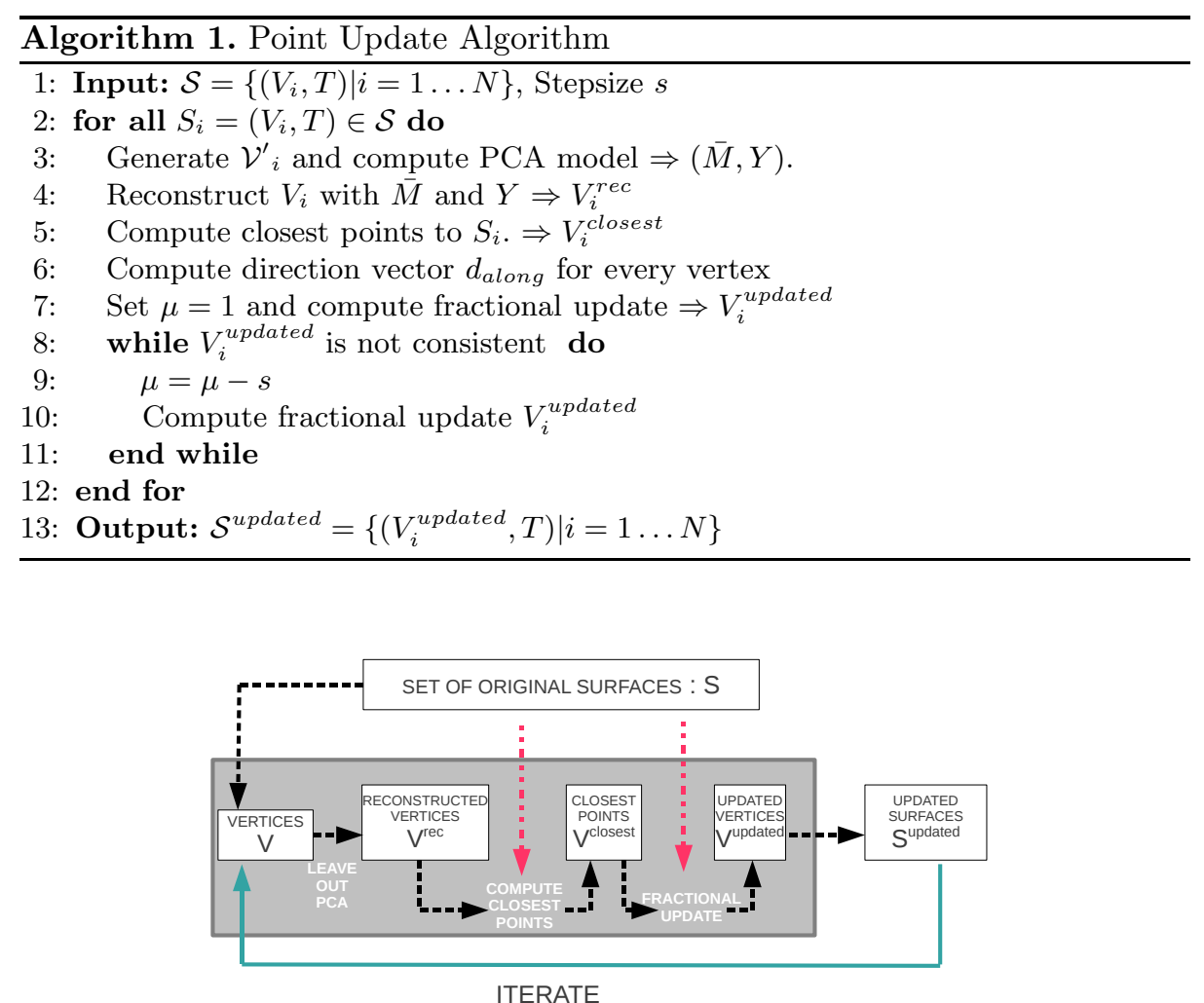

Fig. 2. Algorithm outlining the procedure for iteratively updating points in shape model: A surface is described with a set of vertices and triangles. Vertices are reconstructed using leave out reconstruction. Closest surface points are then computed and a fractional update is applied in the direction of the closest point to generate new vertices. This procedure repeated till convergence.

\section{$3 \quad$ Experimental Results}

\subsection{Data Acquisition and Validation}

We experiment with a pelvis atlas consisting of 110 male patient samples. The data was collected by a physician and anonymized before we performed any processing. Each sample is a segmented 512x512x256 CT volume. Surface meshes (11663 points, 23414 triangles) were extracted for each volume. All meshes were rigidly registered to a template sample to establish initial point correspondences Iteration 0 set. For validation, we performed leave-20 out cross-validation for all the data. We computed the mean vertex error (Distance between original points and reconstructed points), mean surface error (Distance between reconstructed points and original surface) and mean volume error (Sum of surface error and distance from original points to the reconstructed surface (Hausdorff distance)). We also report the standard deviations for all folds of validation. 


\section{$3.2 \quad$ Results}

We first performed leave-20 out cross-validation using initial point correspondences (iteration 0) by varying the number of modes. The black lines in Figure 3 show the average vertex, surface and volume errors for reconstruction with 1-90 modes. Next, we performed the point update (Algorithm 1). The number of modes selected for reconstruction in step 4 was 30 modes since the black line in Figure 3 flattens out after 30 modes. The reconstructed points (step 4) and closest points (step 5) were used to compute the vertex error, the average along surface distance and the average out of surface distance. The maximum and minimum vertex errors were $5.8 \mathrm{~mm}$ and $2.3 \mathrm{~mm}$. The maximum and minimum along surface distances were $5.1 \mathrm{~mm}$ and $1.4 \mathrm{~mm}$. The maximum and minimum out of surface distances were $3.1 \mathrm{~mm}$ and $0.6 \mathrm{~mm}$. Figure 5 shows an example of point update with this first iteration. The full pelvis on top shows the target (original) shape, which is the mesh generated by initial point correspondences for one of the instances. The figures at the bottom show zoomed versions of the some of the regions. The zoomed view on the left shows regions on the target shape and the zoomed view in the middle shows the same mesh with point correspondences updated with the closest points computed. A lot of distortion can be observed by this step. Secondly, some of triangles are flipped with this point update. In order to deal with both of these problems, we performed the adaptive selection of $\mu$ starting with $\mu=1$ and a step size of $s=0.1$. This gives us a $\mu$ value of 0.3 which is the largest value of $\mu$ for generating a consistent dataset. We then performed the leave- 20 out cross-validation of these samples just as in Iteration 0 . The average vertex, surface and volume errors and the error bars are shown in blue in Figure 3. We then demonstrate the effect of $\mu$ on these metrics as follows. We generated datasets for $\mu<0.3$ (consistent datasets) at step sizes of 0.1. This gave us two more datasets at $\mu=0.1$ and $\mu=0.2$. In addition, we also generated one more dataset for $\mu=1$. This dataset was not consistent (at least 30 out of the 23414 triangles were flipped in the meshes). Leave-20 out cross-validation was performed on these three extra datasets. The average vertex, surface and volume errors and error bars are plotted in Figure 3 . The red curves correspond to $\mu=0.1$. The green curves correspond to $\mu=0.2$ and the black dashed curves correspond to $\mu=1$. Thus, we observe that the increase of $\mu$ affects these metrics consistently towards $\mu=1$.

Following this, we performed two more iterations of the leave out reconstruction. Again, we used 30 modes for the reconstruction and $\mu$ was adaptively selected at each subsequent iteration. In iteration $2, \mu$ was selected as 0.3 and at iteration $3, \mu$ was selected as 0.4. Leave-20 out cross-validation was then performed for the same 20 samples and the three metrics are plotted in Figure 4. With validation at 20 modes, the average vertex error drops

Table 1. Vertex, Surface \& Volume Errors, leave-20-out validation, 20 modes

\begin{tabular}{|l|c|c|c|}
\hline & $\begin{array}{c}\text { Vertex } \\
\text { Error(mm) }\end{array}$ & $\begin{array}{c}\text { Surface } \\
\text { Error(mm) }\end{array}$ & $\begin{array}{c}\text { Volume } \\
\text { Error(mm) }\end{array}$ \\
\hline \hline Iter 0 & 4.0 & 1.6 & 3.2 \\
\hline Iter 1 & 2.9 & 1.5 & 2.9 \\
\hline Iter 2 & 2.4 & 1.4 & 2.8 \\
\hline Iter 3 & 2.1 & 1.4 & 2.7 \\
\hline
\end{tabular}



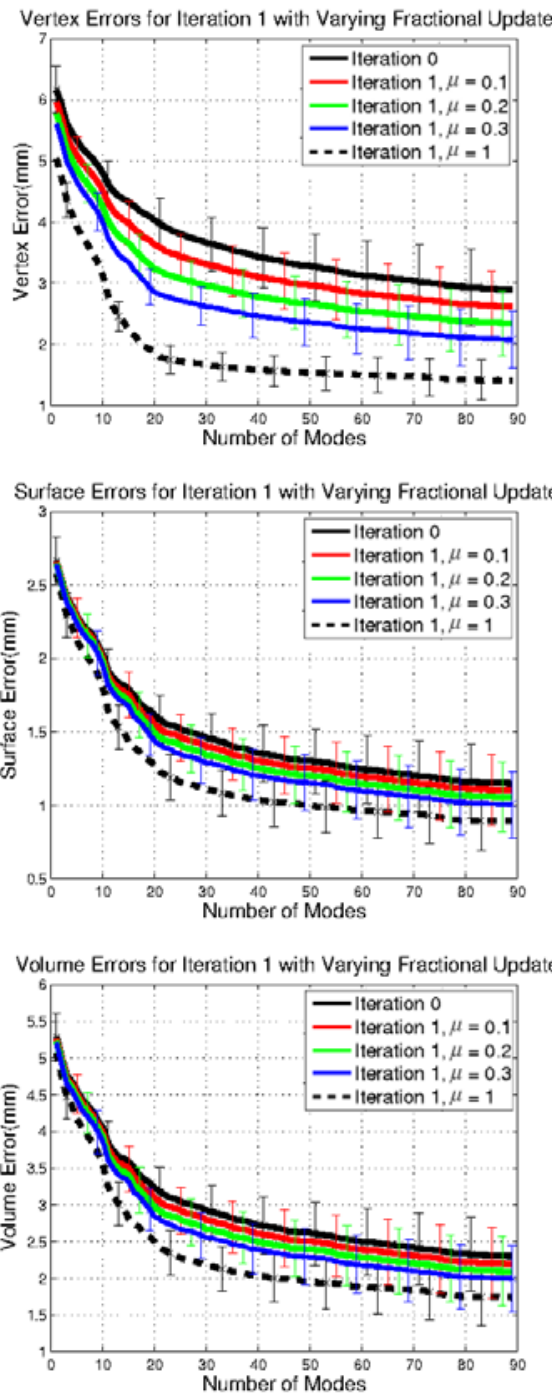

Fig. 3. Graphs showing the Vertex error, Surface error and Volume error with error bars from Leave-20 out crossvalidation for Iteration 1 with varying fractional updates. Leave out reconstruction was performed with 30 modes and values of the fractional updates are $\mu=0.1,0.2$ and 0.3 .
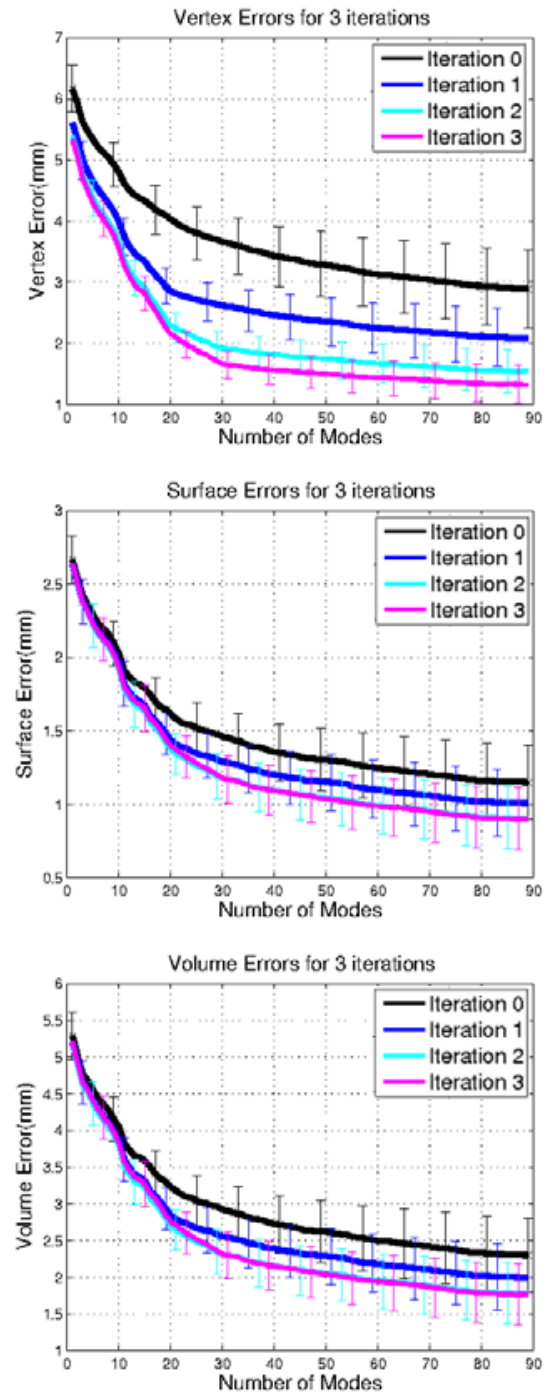

Fig. 4. Graphs showing the Vertex error, Surface error and Volume error with error bars from Leave-20 out crossvalidation for three iterations. Leave out reconstruction was performed with 30 modes. $\mu=$ was adaptively selected as $0.3,0.3$ and 0.4 in iterations 1,2 and 3 respectively. 


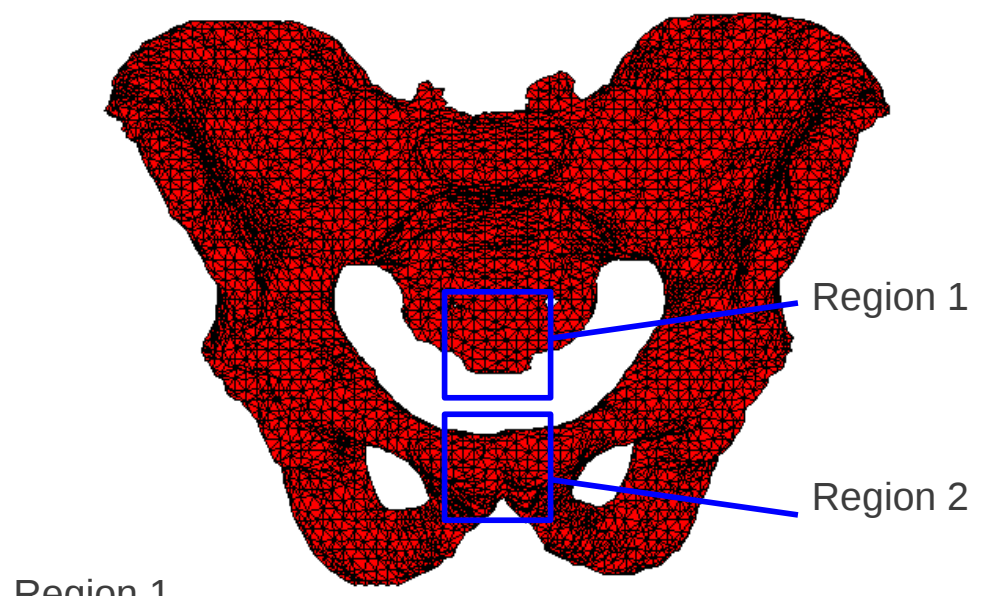

Region 1

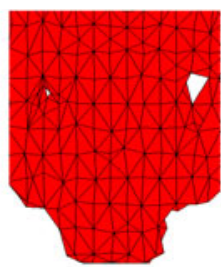

Target Shape

Region 2

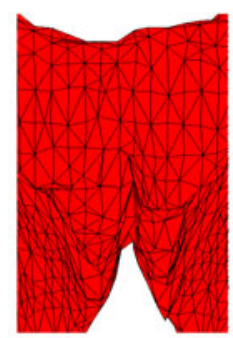

Target Shape

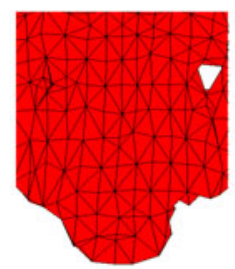

Iteration 1 Closest Points

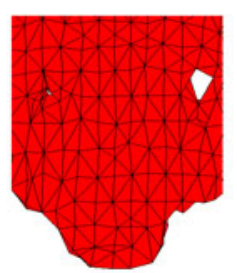
Iteration 3 - Adaptive Fractional Update

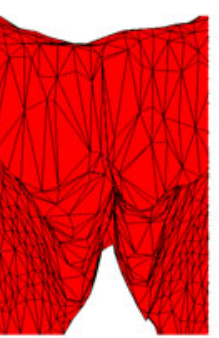

Iteration 1 - Iteration 3 - Adaptive Closest Points

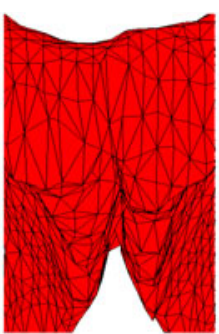

Fractional Update

Fig. 5. Top: Original Mesh of a pelvis instance, Bottom: Zoomed view of mesh in two regions. The left most views show the region on the original pelvis, the middle view shows the region with updates using the closest points in iteration 1 (Black dashed curve in Figure 3), the right most views show the region after iteration 3 with adaptive fractional updates (Pink curve in Figure 4). Note that although the metrics are similar, there is a lot more shape distortion in the closest point reconstruction of iteration 1 . This shows that the fractional update method is also limiting the shape distortions. 
from $4.0 \mathrm{~mm}$ to $2.1 \mathrm{~mm}$, the average surface error drops from $1.6 \mathrm{~mm}$ to $1.4 \mathrm{~mm}$ and the average volume error drops from $3.2 \mathrm{~mm}$ to 2.7 . Table 1 shows these values for all iterations. We also note that the difference between these errors in the last two iterations is very small, showing convergence of the procedure. Finally, we compare the reconstruction of the adaptive fractional updates with the reconstruction with the closest points in iteration 1. By inspection of the graphs in Figures 3 and 4, we note that the closest points in iteration 1 generate the black dashed curve in figure 3 and iteration 3 of the adaptive fractional updates generate the pink curve in Figure 4. The metrics are comparable for these two curves. Figure [5] shows a comparison of the original shape and these two types of point updates. We note that the adaptive fractional update not only maintains consistency of the shape but also reduces the amount of shape distortion.

\section{Discussion}

We have presented a general iterative method for refinement of point 3D point correspondences for statistical atlas modelling. This method is non-specific to the statistical method and does not use outside information to update the point correspondences. Instead, the statistical model's estimate is used to drive the direction of point update. Since our method explicitly computes reconstructions based on the statistical model and then uses the reconstruction to update the points, this method could can be generalized to the use of any statistical model. It is also much faster than previous methods which use intensity based deformable registration or complex optimization procedures. In our experiments, we show that with leave-20 out validation with 20 modes, the vertex error can be reduced from $4 \mathrm{~mm}$ to $2.1 \mathrm{~mm}$, the surface error can be reduced from $1.6 \mathrm{~mm}$ to $1.4 \mathrm{~mm}$ and the volume error can be reduced from $3.2 \mathrm{~mm}$ to $2.7 \mathrm{~mm}$. We also note that the fractional update algorithm not only maintains consistency but also reduces the amount of shape distortion. One of the drawbacks of the current procedure is that no intensity information is used at all. Thus, although the shapes are consistent, it is possible that some of the detail may be lost. The work so far has addressed only one specific atlas. In future work, we plan to extend the evaluation of the applicability of our method to other types of statistical atlases.

Acknowledgments. This work was supported by NIH grant RO1 HD05 5741 (ACE, project IBIS) and by NIH grant U54 EB005149 (NA-MIC).

\section{References}

1. Cootes, T.F., Taylor, C.J., Cooper, D.H., Graham, J.: Active shape models their training and application. Comput. Vis. Image Underst. 61, 38-59 (1995)

2. Chintalapani, G., Ellingsen, L.M., Sadowsky, O., Prince, J.L., Taylor, R.H.: Statistical atlases of bone anatomy: Construction, iterative improvement and validation. In: Ayache, N., Ourselin, S., Maeder, A. (eds.) MICCAI 2007, Part I. LNCS, vol. 4791, pp. 499-506. Springer, Heidelberg (2007) 
3. Davies, R.H., Twining, C.J., Cootes, T.F., Taylor, C.J.: Building 3-d statistical shape models by direct optimization. IEEE Trans. Med. Imaging 29(4), 961-981 (2010)

4. Joshi, S.H., Cabeen, R.P., Sun, B., Joshi, A.A., Gutman, B., Zamanyan, A., Chakrapani, S., Dinov, I., Woods, R.P., Toga, A.W.: Cortical sulcal atlas construction using a diffeomorphic mapping approach. In: Jiang, T., Navab, N., Pluim, J.P.W., Viergever, M.A. (eds.) MICCAI 2010. LNCS, vol. 6361, pp. 357-366. Springer, Heidelberg (2010)

5. Osher, S.J., Fedkiw, R.P.: Level Set Methods and Dynamic Implicit Surfaces, 1st edn. Springer, Heidelberg (2002)

6. Bishop, C.M.: Pattern Recognition and Machine Learning (Information Science and Statistics). Springer-Verlag New York, Inc., Secaucus (2006) 